\title{
Seroepidemiology of cytomegalovirus infection in pregnant women in Durango City, Mexico
}

\author{
Cosme Alvarado-Esquivel ${ }^{1 *}$, Jesús Hernández-Tinoco ${ }^{2}$, Luis Francisco Sánchez-Anguiano², Agar Ramos-Nevárez ${ }^{3}$, \\ Sandra Margarita Cerrillo-Soto³, Sergio Estrada-Martínez², Lucio Martínez-Ramírez³, Alma Rosa Pérez-Álamos² \\ and Carlos Alberto Guido-Arreola ${ }^{3}$
}

\begin{abstract}
Background: Cytomegalovirus causes congenital infections all around the world. The seroepidemiology of cytomegalovirus infection in pregnant women in Mexico is largely unknown. We sought to determine the seroprevalence of cytomegalovirus infection in pregnant women in Durango City, Mexico; and to determine seroprevalence association with socio-demographic, clinical and behavioral characteristics of pregnant women.

Methods: Through a cross-sectional study design, 343 pregnant women were examined for anti-cytomegalovirus IgG and IgM antibodies in Durango City, Mexico. We used a standardized questionnaire to obtain the general characteristics of the pregnant women. Multivariate analysis was performed to determine the association of cytomegalovirus infection with the characteristics of the pregnant women.

Results: Anti-CMV IgG and IgM antibodies were detected in 225 (65.6\%) and in none of the 343 pregnant women studied, respectively. Multivariate analysis showed that CMV exposure was associated with increasing age (OR $=1.67$; 95\% Cl: $1.01-2.76 ; P=0.04)$. Other women characteristics including socioeconomic status, education, blood transfusion, transplantation, sexual promiscuity and number of previous pregnancies or deliveries did not show an association with CMV exposure.

Conclusions: This is the first seroepidemiology study of CMV infection in pregnant women in Mexico. A number of known factors associated with CMV infection were not associated with CMV exposure in the women studied. Further studies to determine routes of CMV infection in pregnant women in Mexico are needed.
\end{abstract}

Keywords: Cross-sectional study, Seroprevalence, Cytomegalovirus, Infection, Epidemiology, Mexico

\section{Background}

Cytomegalovirus (CMV) is a DNA virus of the Betaherpesvirinae subfamily and Herpesviridae family [1]. CMV is a leading cause of congenital infections all around the world [2-4]. Congenital infections with CMV may be asymptomatic [5] or may lead to hearing impairment, mental retardation, cerebral palsy [6], and neurodevelopmental disabilities [2,4]. The incidence of congenital CMV infection is about $1 \%-7 \%$ of births [4]. In addition, congenital CMV infection may occur not only in primary but also in non-primary maternal infections [4]. Infections

\footnotetext{
* Correspondence: alvaradocosme@yahoo.com

'Biomedical Research Laboratory, Faculty of Medicine and Nutrition, Juárez University of Durango State, Avenida Universidad S/N, 34000 Durango, Mexico

Full list of author information is available at the end of the article
}

with CMV persist through chronic and latent states of infections [7], and can be reactivated with shedding of infectious virus $[1,8]$. Transmission of CMV occurs by person-to-person contact [1]. In addition, CMV infection acquired by blood transfusion may lead to significant complications in immunocompromised individuals [9]. Severe disease including pneumonia, retinitis, hepatitis, encephalitis, and other organ involvements due to CMV in immunocompromised patients have been reported [10]. Very little is known about the seroepidemiology of CMV infection in Mexican populations. We are not aware of any study on the seroepidemiology of CMV infection in pregnant women in Mexico. Therefore, we sought to determine the seroprevalence of CMV infection in pregnant women in Durango City, Mexico. In addition, the socio-demographic, clinical and behavioral 
characteristics of pregnant women associated with CMV infection were also investigated.

\section{Methods}

\section{Study population and study design}

Through a cross-sectional study design, we studied pregnant women in a public primary health care center (Clínica de Medicina Familiar, Instituto de Seguridad y Servicios Sociales de los Trabajadores del Estado) in Durango City, Mexico from April to November 2013. Inclusion criteria for enrollment in the study were: 1) pregnant women; 2) residing in Durango City; 3) aged 15 years and older; and 4) who accepted to participate in the study.

\section{Socio-demographic, clinical and behavioral data}

We submitted a standardized questionnaire to obtain socio-demographic, clinical and behavioral characteristics from the pregnant women studied. Socio-demographic data included age, birthplace, residence, occupation, and educational and socio-economic status. Clinical data included obstetric history (month of pregnancy, number of pregnancies, deliveries, cesarean sections and miscarriages), presence of any underlying disease, history of lymphadenopathy, frequent headaches and impairments of memory, reflexes, vision and hearing, and history of hepatitis, blood transfusions, transplants or surgery. Behavioral data included addictions, sexual promiscuity, washing hands before eating, eating away of home (in restaurants and fast food outlets), consumption of unpasteurized milk, untreated water, and unwashed raw vegetables or fruits, foreign travel, and type of flooring at home.

\section{Laboratory tests}

Serum samples were obtained from each pregnant women by centrifugation of whole blood. Sera of the women were kept frozen until analyzed. Sera were examined for anti-CMV IgG antibodies by a commercially available enzyme immunoassay "Cytomegalovirus IgG (CMV IgG)” kit (Diagnostic Automation Inc., Calabasas, CA, USA) and for anti-CMV IgM antibodies by a commercially available enzyme immunoassay "Cytomegalovirus IgM (CMV IgM" kit (Diagnostic Automation Inc., Calabasas, CA, USA). The tests were performed following the instructions of the manufacturer. The cut-off values for IgG and IgM seropositivity were obtained as follows: firstly, the mean optical densities of $\operatorname{IgG}$ and IgM calibrators were multiplied by the correction factor (0.50-0.55) printed on the label of calibrators to obtained the corrected mean cut-off value: then, we calculated the CMV G and $\mathrm{M}$ indexes by dividing the optical density of each sample by the corrected mean cut-off value. A sample was considered positive for IgG or IgM when a CMV G index or a CMV M index was greater than 1.1, respectively. These assays are qualitative, and no quantification of antibody levels were performed.

\section{Ethical aspects}

This study was approved by the ethical committee of the Instituto de Seguridad y Servicios Sociales de los Trabajadores del Estado in Durango City. The purpose and procedures of the study were explained to all pregnant women, and a written informed consent was obtained from all of them and from the next of kin of minor participants.

\section{Statistical analysis}

The statistical analysis was performed with the aid of the software: Epi Info version 7 and SPSS version 15.0. For calculation of the sample size, we used a value of 15,000 as a population size from which the sample was selected, a reference seroprevalence of $89.2 \%$ [11] as expected frequency of the factor under study, $3.5 \%$ of confidence limits, a design effect of 1.0, one cluster, and a confidence level of $95 \%$. The result of the calculation was 296 subjects. We used the Pearson's chi square and the Fisher exact test (when values were small) for comparison of the frequencies among groups. Bivariate and multivariate analyses were used to evaluate the association between the characteristics of the women and CMV seropositivity. Variables with a $P$ value equal to or less than 0.10 obtained in the bivariate analysis were included in the multivariate analysis. Odd ratios (OR) and 95\% confidence intervals (CI) were calculated by multivariate analysis using the Enter method. A $P$ value less than 0.05 was considered statistically significant.

\section{Results}

In total, we enrolled 343 pregnant women. Most of them were born in Durango; their mean age was $28.88 \pm 6.12$ years (range 15-43 years). General socio-demographic and obstetric characteristics of the pregnant women studied are shown in Table 1. Anti-CMV IgG antibodies were detected in 225 $(65.6 \%)$ of the 343 pregnant women studied. None of the 343 pregnant women had anti-CMV IgM antibodies.

Of the socio-demographic characteristics of the pregnant women (Table 1), only the variable age showed a $P$ value $<0.10$ by bivariate analysis. Other socio-demographic characteristics including birthplace, residence, educational level, occupation and socio-economic status had $P$ values $>0.10$ by bivariate analysis.

With respect to behavioral characteristics, smoking was the only variable that showed a $P$ value $<0.10$ by bivariate analysis. Other behavioral characteristics including foreign travel, eating away of home, consumption of untreated water, and unwashed raw vegetables or fruits, drug or alcohol addictions, sexual promiscuity, washing hands before eating, and type of flooring at home show $P$ values $>0.10$ by bivariate analysis. 
Table 1 Socio-demographic and obstetric characteristics of pregnant women and seroprevalence of $\mathrm{CMV}$ infection

\begin{tabular}{llll}
\hline & $\begin{array}{c}\text { No. of } \\
\text { women }\end{array}$ & $\begin{array}{c}\text { Prevalence of CMV } \\
\text { infection }\end{array}$ & P value \\
\cline { 3 - 3 } Characteristic & tested $^{\mathrm{a}}$ & No. & $\%$ \\
\hline
\end{tabular}

Age groups (years)

$\begin{array}{lllll}15-30 & 198 & 119 & 60.1 & 0.009 \\ 31-43 & 144 & 106 & 73.6 & \end{array}$

Birth place

Durango State

Other Mexican State

319

20

209

65.5

0.68

Residence place

Durango State

$$
342
$$

Other Mexican State

Residence area

Urban

Rural

Educational level

Up to 6 years

7-12 years

13 or more years

Occupation

\section{Unemployed $^{b}$}

Employed $^{c}$

Socio-economic level

Low
Medium
High
Month of pregnancy

1 to 3
4 to 6
7 to 9
Deliveries

\begin{tabular}{|c|c|c|c|c|}
\hline Yes & 140 & 94 & 67.1 & 0.59 \\
\hline No & 202 & 130 & 64.4 & \\
\hline \multicolumn{5}{|l|}{ Pregnancies } \\
\hline 3 or more & 228 & 158 & 69.3 & 0.04 \\
\hline 1 or 2 & 115 & 67 & 58.3 & \\
\hline \multicolumn{5}{|c|}{ Cesarean sections } \\
\hline None & 241 & 155 & 64.3 & 0.47 \\
\hline 1 or 2 & 101 & 69 & 68.3 & \\
\hline \multicolumn{5}{|l|}{ Miscarriages } \\
\hline None & 269 & 173 & 64.7 & 0.37 \\
\hline $1-3$ & 73 & 51 & 69.9 & \\
\hline
\end{tabular}

apregnant women with available data.

bUnemployed = none occupation, student or housewife.

${ }^{\mathrm{C}}$ Employed = Employee, professional, business, or other.
Concerning clinical characteristics, seroprevalence of CMV infection was similar in ill $(7 / 15: 46.7 \%)$ than in healthy (217/327: 66.4\%) pregnant women $(P=0.11)$. In contrast, seroprevalence of $\mathrm{CMV}$ infection was significantly higher $(P=0.04)$ in pregnant women with history of lymphadenopathy (39/50: 78.0\%) than in those without such history (186/292: 63.7\%). Pregnant women with more than two pregnancies had a significantly higher $(P=0.04)$ seroprevalence of CMV exposure (158/228: 69.3\%) than those with one or two pregnancies (67/115: 58.3\%). Other clinical characteristics of pregnant women including impairments of memory, reflexes, vision and hearing, history of hepatitis, blood transfusions, transplants or surgery, and other obstetric characteristics (month of pregnancy, number of deliveries, cesarean sections and miscarriages) were not associated with CMV infection.

Multivariate analysis of socio-demographic, clinical and behavioral variables with $P$ values equal to or lower than 0.10 by bivariate analysis (Table 2) showed that CMV infection was positively associated only with age $(\mathrm{OR}=1.67 ; 95 \% \mathrm{CI}: 1.01-2.76 ; P=0.04)$.

\section{Discussion}

There is a lack of knowledge about the epidemiology of CMV infection in pregnant women in Mexico. The present study was performed to investigate the seroprevalence and correlates of CMV infection in pregnant women in the northern Mexican city of Durango. We found a $65.6 \%$ seroprevalence of CMV infection in the pregnant women studied. Based on the seropositivity to CMV IgG and IgM antibodies, all women with CMV exposure had latent infection and none had recent or acute CMV infection. We are not aware of previous reports about the seroepidemiology of CMV infection in pregnant women in Mexico. Therefore, we cannot compare our seroprevalence results with others in pregnant women in Mexico. In a study in healthy women of reproductive age in Cuernavaca City in central Mexico, researchers found a 92.6\% seroprevalence of CMV infection [12]. While in a national survey in subjects aged 1 to 70 years old in Mexico an 88.2\% seroprevalence of CMV infection was found [11]. On the other hand, comparison of our seroprevalence with those reported in

Table 2 Multivariate analysis of selected characteristics of pregnant women and their association with CMV infection

\begin{tabular}{lccc}
\hline Characteristic & Odds ratio $^{\mathbf{a}}$ & $\begin{array}{c}\mathbf{9 5 \%} \text { confidence } \\
\text { interval }\end{array}$ & $\boldsymbol{P}$ value \\
\hline Age (31-43 years) & 1.67 & $1.01-2.76$ & 0.04 \\
Lymphadenopathy & 2.03 & $0.98-4.19$ & 0.05 \\
More than 2 pregnancies & 1.36 & $0.81-2.26$ & 0.23 \\
Smoking & 0.53 & $0.26-1.07$ & 0.08 \\
\hline
\end{tabular}

${ }^{a}$ Adjusted by the characteristics included in this Table. 
other countries suggests that the seroprevalence of CMV infection in pregnant women in Durango could be placed in an intermediate position of endemicity. The $65.6 \%$ seroprevalence found in pregnant women in Durango is similar to the $62.4 \%-66 \%$ seroprevalences reported in pregnant women in Poland [13], Japan [14], and Norway [15], and higher than the 49\% seroprevalence in white British pregnant women in the United Kingdom [16] and a 42.3\% seroprevalence in pregnant women in Germany [17]. In contrast, the seroprevalence found in pregnant women in Durango is lower than the $92.6 \%-100 \%$ seroprevalences reported in pregnant women in Iran [18], Palestine [19], Brazil [20], Turkey [21], Nigeria [22], and Cuba [23].

Analysis of the socio-demographic characteristics showed that the seroprevalence of CMV infection in pregnant women increased with age. This finding is consistent with those reported in other studies where researchers found an increase in the seroprevalence of CMV infection with age in both pregnant women [13] and general population [24]. Other known contributing factors for CMV infection including socioeconomic status [8], education, sexual promiscuity [12], blood transfusion and transplantation [25] did not show any association with CMV exposure in the women studied. Of the sources of infection with CMV in pregnant women stands out young children [26,27]. We assess the contact with young children by evaluating the number of children the women have already born (history of pregnancies), considering that women with more than two pregnancies had a higher contact with children than women with fewer pregnancies. Analysis showed that women with more than two pregnancies had a higher, but not statistically significant, seroprevalence of CMV infection than those with one or two pregnancies. This approach was a limitation of the study since contact with children can occur not only at home but also at work in some women. In the present study, the occupation of housewife was not associated with CMV exposure. None of the pregnant women studied reported to be schoolteacher or baby-sitter and the lack of these occupations among the studied women might have a negative influence on the seroprevalence. Therefore, the association of CMV exposure with contact with children should be further evaluated in pregnant women in Mexico. Hygiene practices are important in the prevention of CMV infection [26]. However, in this study the variable washing hands before eating did not show an association with CMV exposure.

\section{Conclusions}

This is the first seroepidemiology study of CMV infection in pregnant women in Mexico. A number of known factors associated with CMV infection were not associated with CMV exposure in the women studied. Further studies to determine routes of CMV infection in pregnant women in Mexico are needed.
Competing interests

The authors declare that they have no competing interests.

\section{Authors' contributions}

CAE conceived and designed the study protocol, performed the laboratory tests, analyzed the data, and wrote the manuscript. JHT and LFSA performed the data analysis and wrote the manuscript. ARN, SMCR and LSS obtained the blood samples and clinical data, and performed the data analysis. SEM performed the statistical analysis. ARPA performed the laboratory tests. All authors read and approved the final version of the manuscript.

\section{Acknowledgment}

This study was financially supported by Juarez University of Durango State.

\section{Author details}

${ }^{1}$ Biomedical Research Laboratory, Faculty of Medicine and Nutrition, Juárez University of Durango State, Avenida Universidad S/N, 34000 Durango, Mexico. ${ }^{2}$ Institute for Scientific Research "Dr. Roberto Rivera-Damm", Juárez University of Durango State, Avenida Universidad S/N, 34000 Durango, Mexico. ${ }^{3}$ Clínica de Medicina Familiar, Instituto de Seguridad y Servicios Sociales de los Trabajadores del Estado, Predio Canoas S/N, 34079 Durango, Mexico.

Received: 5 July 2014 Accepted: 3 September 2014

Published: 5 September 2014

\section{References}

1. de Jong MD, Galasso GJ, Gazzard B, Griffiths PD, Jabs DA, Kern ER, Spector SA: Summary of the II International Symposium on Cytomegalovirus. Antiviral Res 1998, 39:141-162.

2. Hyde TB, Schmid DS, Cannon MJ: Cytomegalovirus seroconversion rates and risk factors: implications for congenital CMV. Rev Med Virol 2010, 20:311-326. doi:10.1002/rmv.659.

3. Kenneson A, Cannon MJ: Review and meta-analysis of the epidemiology of congenital cytomegalovirus (CMV) infection. Rev Med Virol 2007, $17: 253-276$

4. Manicklal S, Emery VC, Lazzarotto T, Boppana SB, Gupta RK: The "silent" global burden of congenital cytomegalovirus. Clin Microbiol Rev 2013, 26:86-102. doi:10.1128/CMR.00062-12.

5. Buonsenso D, Serranti D, Gargiullo L, Ceccarelli M, Ranno O, Valentini P: Congenital cytomegalovirus infection: current strategies and future perspectives. Eur Rev Med Pharmacol Sci 2012, 16:919-935.

6. Swanson EC, Schleiss MR: Congenital cytomegalovirus infection: new prospects for prevention and therapy. Pediatr Clin North Am 2013, 60:335-349. doi:10.1016/j.pcl.2012.12.008.

7. Goodrum F, Caviness K, Zagallo P: Human cytomegalovirus persistence. Cell Microbiol 2012, 14:644-655. doi:10.1111/j.1462-5822.2012.01774.x.

8. Cannon MJ, Schmid DS, Hyde TB: Review of cytomegalovirus seroprevalence and demographic characteristics associated with infection. Rev Med Virol 2010, 20:202-213. doi:10.1002/rmv.655.

9. Lindholm PF, Annen K, Ramsey G: Approaches to minimize infection risk in blood banking and transfusion practice. Infect Disord Drug Targets 2011, 11:45-56.

10. Foti G, Hyeraci M, Kunkar A, leropoli G, Sofo D, De Lorenzo S, Carpentieri MS: Cytomegalovirus infection in the adult. Minerva Med 2002, 93:109-117.

11. Conde-Glez C, Lazcano-Ponce E, Rojas R, DeAntonio R, Romano-Mazzotti L, Cervantes Y, Ortega-Barria E: Seroprevalences of varicella-zoster virus, herpes simplex virus and cytomegalovirus in a cross-sectional study in Mexico. Vaccine 2013, 31:5067-5074. doi:10.1016/j.vaccine.2013.08.077.

12. Echániz-Avilés G, Tamayo-Legorreta E, Cruz-Valdez A, Rangel-Flores H, Hernández-Nevárez P, Gatica-Marquina R, Calderón-Jaimes E: Prevalence of antibodies against cytomegalovirus in women of reproductive age. Salud Publica Mex 1993, 35:20-26.

13. Wujcicka W, Gaj Z, Wilczyński J, Sobala W, Spiewak E, Nowakowska D: Impact of socioeconomic risk factors on the seroprevalence of cytomegalovirus infections in a cohort of pregnant Polish women between 2010 and 2011. Eur J Clin Microbiol Infect Dis 2014, in press.

14. Taniguchi K, Watanabe N, Sato A, Jwa SC, Suzuki T, Yamanobe Y, Sago H, Kozuka K: Changes in cytomegalovirus seroprevalence in pregnant 
Japanese women-a 10-year single center study. J Clin Virol 2014, 59:192-194. doi:10.1016/j.jcv.2013.12.013.

15. Barlinn R, Vainio K, Samdal HH, Nordbø SA, Nøkleby H, Dudman SG: Susceptibility to cytomegalovirus, parvovirus B19 and age-dependent differences in levels of rubella antibodies among pregnant women. J Med Virol 2014, 86:820-826. doi:10.1002/jmv.23757.

16. Pembrey L, Raynor P, Griffiths P, Chaytor S, Wright J, Hall AJ: Seroprevalence of cytomegalovirus, Epstein Barr virus and varicella zoster virus among pregnant women in Bradford: a cohort study. PLoS One 2013, 8:e81881. doi:10.1371/journal.pone.0081881.

17. Enders $G$, Daiminger A, Lindemann L, Knotek F, Bäder U, Exler S, Enders M: Cytomegalovirus (CMV) seroprevalence in pregnant women, bone marrow donors and adolescents in Germany, 1996-2010. Med Microbiol Immunol 2012, 201:303-309. doi:10.1007/s00430-012-0232-7.

18. Erfanianahmadpoor M, Nasiri R, Vakili R, Hassannia T: Seroprevalence, transmission, and associated factors of specific antibodies against cytomegalovirus among pregnant women and their infants in a regional study. Saudi Med J 2014, 35:360-364.

19. Neirukh T, Qaisi A, Saleh N, Rmaileh AA, Zahriyeh EA, Qurei L, Dajani F, Nusseibeh T, Khamash H, Baraghithi S, Azzeh M: Seroprevalence of cytomegalovirus among pregnant women and hospitalized children in Palestine. BMC Infect Dis 2013, 13:528. doi:10.1186/1471-2334-13-528.

20. Yamamoto AY, Castellucci RA, Aragon DC, Mussi-Pinhata MM: Early high CMV seroprevalence in pregnant women from a population with a high rate of congenital infection. Epidemiol Infect 2013, 141:2187-2191. doi:10.1017/S0950268812002695.

21. Uysal A, Taner CE, Cüce M, Atalay S, Göl B, Köse S, Uysal F: Cytomegalovirus and rubella seroprevalence in pregnant women in Izmir/Turkey: follow-up and results of pregnancy outcome. Arch Gynecol Obstet 2012, 286:605-608. doi:10.1007/s00404-012-2353-z.

22. Akinbami AA, Rabiu KA, Adewunmi AA, Wright KO, Dosunmu AO, Adeyemo TA, Adediran A, Osunkalu VO: Seroprevalence of cytomegalovirus antibodies amongst normal pregnant women in Nigeria. Int J Womens Health 2011 3:423-428. doi:10.2147/JWH.S24850.

23. Correa CB, Kourí V, Verdasquera D, Martínez PA, Alvarez A, Alemán Y, Pérez L, Viera J, González R, Pérez E, Moro I, Navarro MA, Melin P: HCMV seroprevalence and associated risk factors in pregnant women, Havana City, 2007 to 2008. Prenat Diagn 2010, 30:888-892. doi:10.1002/pd.2587.

24. Staras SA, Dollard SC, Radford KW, Flanders WD, Pass RF, Cannon MJ: Seroprevalence of cytomegalovirus infection in the United States, 1988-1994. Clin Infect Dis 2006, 43:1143-1151.

25. Raynor BD: Cytomegalovirus infection in pregnancy. Semin Perinatol 1993 17:394-402.

26. Johnson J, Anderson B, Pass RF: Prevention of maternal and congenital cytomegalovirus infection. Clin Obstet Gynecol 2012, 55:521-530. doi:10.1097/GRF.0b013e3182510b7b

27. Cannon MJ, Hyde TB, Schmid DS: Review of cytomegalovirus shedding in bodily fluids and relevance to congenital cytomegalovirus infection. Rev Med Virol 2011, 21:240-255. doi:10.1002/rmv.695.

doi:10.1186/1471-2334-14-484

Cite this article as: Alvarado-Esquivel et al:: Seroepidemiology of cytomegalovirus infection in pregnant women in Durango City, Mexico. BMC Infectious Diseases 2014 14:484

\section{Submit your next manuscript to BioMed Central and take full advantage of:}

- Convenient online submission

- Thorough peer review

- No space constraints or color figure charges

- Immediate publication on acceptance

- Inclusion in PubMed, CAS, Scopus and Google Scholar

- Research which is freely available for redistribution

Submit your manuscript at www.biomedcentral.com/submit
C Biomed Central 